Participants 42,054 adult U.S. players (3,481 teams and 7,673 Rugby-7s matches).

Assessment of Risk Factors Match injury rates, site, type, and severity.

Main measurement outcome Incidence (per 1000 player-hour (ph)), severity and details of mechanisms were captured using Rugby Injury Survey \& Evaluation (RISE) report methodology. All-injuries (medical-attention and time-loss) and time-loss injuries were defined. Days (d) absent before return-to-training/ competition (including post-tournament) were recorded.

Results A total of 498 HNF injuries were encountered (allinjury rate $=16.7 / 1000 \mathrm{ph} ; \operatorname{men}=17.3 / 1000 \mathrm{ph} ; \quad$ women $=15.5 /$ 1000ph; IRR:1.1; CI:0.9-1.4). Head/face was the most commonly injured site (all-injuries $=90.8 \%$; time-loss $=86.8 \%$ ) followed by neck/cervical spine (all-injuries $=9.2 \%$; timeloss $=13.2 \%)$. Concussions were the most common type of injury $(36.7 \% ; 6.1 / 1000 \mathrm{ph})$ followed by facial lacerations $(28.7 \% ; 4.8 / 1000 \mathrm{ph})$. Incidence of concussions was similar between sexes (IRR:1.0; CI:0.7-1.3). Longer return-to-sport were seen among women $(46.4 \mathrm{~d})$ than men $(32.1 \mathrm{~d} ; P=0.047)$ post-concussion. Scalp/face lacerations were more common among men than women (IRR:2.5; CI:1.6-4.1; $P<0.001$ ). Time-loss injuries occurred similarly among men and women (IRR:1.5; CI:1.0-2.6; $P=0.061)$. HNF injury severity was similar between sexes $(29.3 \pm 32.4$ days absent from play). The tackle $(71.5 \%)$ was the most common injury event. Men sustained HNF injuries mostly with direct contact with another player (IRR:1.3; CI:1.0-1.7; $P=0.023$ ), while women were injured with impact with the playing surface (IRR:1.8; CI:1.0$3.2 ; P=0.032$ ).

Conclusions Incidence of HNF injuries were similar between sexes among U.S. rugby-7s players. Sex differences with concussion severity and contact mechanism of HNF injuries were seen between sexes. Recognition of HNF injury patterns and sex differences will allow for a more effective injury prevention plan in this emerging U.S. collision sport.

\section{NEW ZEALAND SUPER RUGBY INJURY SURVEILLANCE: MATCH INJURIES FROM 2015-2018}

${ }^{1}$ Danielle Salmon, ${ }^{1}$ Ian Murphy, ${ }^{1}$ Kenneth Quarrie, ${ }^{6}$ Greg MacLeod, ${ }^{6}$ Asheer Singh, ${ }^{6}$ Adam Letts, ${ }^{2} J o h n$ Roche, ${ }^{2}$ Deborah Robinson, ${ }^{2}$ Martin Swan, ${ }^{3}$ Theo Dorfling, ${ }^{3}$ Cameron Shaw, ${ }^{4}$ Katherine Rottier, ${ }^{4}$ Kevin Bell, ${ }^{5}$ Stephen Kara, ${ }^{5}$ James McGarvey. ${ }^{1}$ New Zealand Rugby, Wellington, New Zealand; ${ }^{2}$ Crusaders Super Rugby Franchise, Christchurch, New Zealand; ${ }^{3}$ Hurricanes Super Rugby Franchise, Wellington, New Zealand; ${ }^{4}$ Chiefs Super Rugby Franchise, Hamilton, New Zealand; ${ }^{5}$ Blues Super Rugby Franchise, Auckland, New Zealand; ${ }^{6}$ Highlanders Super Rugby Franchise, Dunedin, New Zealand

\subsection{6/bjsports-2021-IOC.229}

Background Rugby union is a collision-based sport, as such the occurrence of injuries resulting in time-loss from participation is inevitable. In 2015 New Zealand Rugby (NZR) began an injury surveillance program with all NZ-based Super Rugby teams to capture all time-loss injuries.

Objective To examine the incidence, burden, severity, nature and cause of match injuries in NZR Super Teams.

Design A prospective observational study.

Setting Match injuries sustained during the 2015-2018 Super rugby seasons.

Patients (or Participants) All contracted rugby players $(n=190)$ over the four seasons.

Main Outcome Measurements Primary outcomes of interest were incidence (injuries/1000 player match-hours), injury burden (days absence/1000 player-match-hours), median severity (days absence), location (\%), diagnosis (\%) and mechanism (\%).

Results From 2015-2018 the following incidence was documented 122, 85, 94 and 81 injuries/1000 player-match-hours respectively. During the first year the initial injury incidence was 122 injuries/1000 player-match-hours, which was significantly higher than 2018, 81 injuries/1000 player-match-hours. The days absence increased over the period from a median of 7 days missed in 2015 (burden: 2262 days absence/1000 player-match-hours) to 16 in 2018 (burden: 3206 days absence/1000 player-match-hours). There was a significant difference in the incidence of injury by position, where forwards sustained 106 injuries/1000 player-match-hours and backs 85 . The most at risk position was the hooker (118 injuries/1000 player-match-hours) and the lowest inside backs (59 injuries/ 1000 player-match-hours). Tackling was the most common mechanism of injury 27\%, followed by being tackled $25 \%$ and collisions $12 \%$. The head was the most common injury location (19\%), followed by the shoulder (13\%) and knee $(10 \%)$. The three most commonly diagnosed injuries were sprains (28\%), concussions (20\%) and muscle rupture/strains/ tear/cramp (16\%).

Conclusions The current data supports the international trend in professional rugby where the time-loss associated with each injury is increasing, resulting in higher levels of injury burden.

\section{A STUDY ON PREVALENCE AND RISK FACTORS ASSOCIATED WITH INJURIES OF INTER-UNIVERSITY RUGBY PLAYERS IN SRI LANKA BASED ON PLAYING POSITION}

Pasindu Silva. University of Sri Jayewardenepura, Colombo, Sri Lanka

\subsection{6/bjsports-2021-IOC.230}

Background Sri Lankan university rugby players only have 3-4 years to understand the game and master its techniques. Due to the nature of the game, players can be seriously injured without proper skills.

Objective This study aimed to understand the injury patterns of University-level rugby players according to their positions.

Design This prospective study of university-level rugby players was carried out after the Sri Lanka University Games (SLUG) 2019 concluded.

Setting The study population was players in the squads of universities that participated in SLUG 2019, which is considered an amateur rugby tournament.

Patients (or Participants) The participants who volunteered were screened with the following inclusion criteria: 1. Age range: $23 \pm 3$ years, 2. Registered for SLUG 2019, 3. Injuryfree for a window of 6 months before the start of the season, 4. Free from systemic injuries

Interventions (or Assessment of Risk Factors) The study examined which player positions are more prone to injuries. Accordingly, the risk factors identified were: contact injury, contact event, injury location and injury type.

Main Outcome Measurements Significant associations between the player position and the above-mentioned risk factors were explored.

Results The most injury-prone position was the Lock position $(18.4 \%)$ whereas the least injury-prone positions were Fly- 